\title{
INTEGRATED BATCH PLANNING OPTIMIZATION BASED ON FUZZY GENETIC AND CONSTRAINT SATISFACTION FOR STEEL PRODUCTION
}

\author{
Su, L. ${ }^{* * *, \#}$; Qi, Y. ; Jin, L.-L.** \& Zhang, G.-L.** \\ *School of Electronics and Information Engineering, Xi'an Jiaotong University, Xi'an 710049, China \\ ${ }^{* *}$ School of Mathematics and Statistics, Hainan Normal University, Haikou 571158, China \\ E-Mail: sulijt@stu.xjtu.edu.cn ( ${ }^{\#}$ Corresponding author)
}

\begin{abstract}
This paper stablishes the model of the integrated batch planning process, and proposes an improved algorithm for this problem. The simulation results of a computerized scheduling system are given to prove the fitness of the model. The steel making casting production process scheduling problem is very difficult to get a good performance solution in practice. The scheduling of steelmaking casting production is a complicated problem of combinational optimization in the hybrid flow shop, which is an NP-Hard problem, and determining the polynomial time algorithm to arrive at the accurate optimal solution has proved to be a difficult task. An improved fuzzy genetic optimization and improved algorithm strategy are proposed. The results show that the method is very efficient in solving the steel casting production scheduling problem.

(Received, processed and accepted by the Chinese Representative Office.)
\end{abstract}

Key Words: Integrated Batch Planning, Scheduling Problem, Fuzzy Genetic

\section{INTRODUCTION}

The iron and steel industry is a vital foundation of national economy, and China is the world's largest manufacturer of steel products. However, since it is a high energy-consuming industry and energy costs have sharply increased in recent years, the problem of how to effectively save energy has attracted increasing attention within the industry. To overcome the obstacle of increasing fuel costs, the global iron and steel industry has experienced a revolution to promote improved production equipment and technologies. To advance its core competence, the industry should take measures to deal with the challenges of cost reduction, quality promotion, and investment decisions, while production planning and coordination are the most crucial aspects in production management.

The challenge of achieving optimal scheduling in steelmaking casting production has continuously been the priority and has drawn great attention from the industry. A reasonable scheduling plan can effectively reduce production cost and energy consumption, promote quality and efficiency and reduce the emission of carbon and other pollutants. Bellabdaoui and Teghem identify a mixed integer linear programming model to find the solution to the continuous casting problem [1]. However, the optimization algorithm cannot arrive at the optimal solution to scheduling in steelmaking casting production in a timely manner. However, heuristic algorithm and meta-heuristic algorithm can meet this expectation in time. Pan et al. propose to use the integer linear programming model to describe the time relationship between furnace and machines [2]. Through using the heuristic method and various improvement strategies to optimize the scheduling results as well as using the artificial bee colony algorithm to adjust the sequence of changing the furnace. Atighehchian et al. suggest manipulating the ant colony algorithm and nonlinear optimization to solve the problem [3]. Liu and Sun study the batch split strategy of optimal scheduling in steelmaking casting production [4]. Pacciarelli and Pranzo analyse the feasible condition of the model and 
the time relationship among all scheduling furnace units, and find the mass scheduling results through using the beam search algorithm [5]. Li et al. develop the classic model and argue that every stage has two machines and used the rolling horizon scheduling algorithm to adjust and test [6].

Utilizing the production method of integrating direct hot charge rolling and direct rolling process as well as systematically composing three traditional steel production elements, steelmaking, continuous casting and hot rolling, has been the absolute choice in the modern steel industry to realize volume production [7, 8]. The current studies can be divided into three types according to the integration plan strategies: (1) the push type strategy depends on a forward process; that is, following the sequence from steelmaking, continuous casting to hot rolling to make the furnace schedule, casting schedule and rolling schedule [9, 10]. (2) The pull type strategy depends on a reverse process; that is, following the reverse sequence from hot rolling, continuous casting to steel-making to make the furnace schedule, casting schedule and rolling schedule [11-13]. (3) The mixed strategy initially follows the pull strategy to make the scheduling and then the push strategy to coordinate [14-17].

Li et al. [6] proposed a unit-specific event for the steelmaking continuous casting scheduling process, and Lin et al. [18] proposed a continuous optimal approach for the mid-size steelmaking continuous casting problem. Then this approach was extended to a large scale industrial batch plant $[19,20]$. Chen et al. [21] improved the Lagrange Relaxation approach for the manufacturing job shop scheduling problems, and Fisher [22] employed this method to solve the integer programming production problems. More recently, Tang et al. [23] established a mathematical program model embedded within the LR to address the SSC production scheduling problem. Almost at the same time, the subgradient method and the bundle method were proposed for job shop scheduling problems [24, 25].

From the observation, however, all the algorithms mentioned above cannot solve the actual steelmaking casting production process scheduling problems. Form all the previous methods, even employing highly complex algorithms and modern super computers, the computation time used to solve the problems of integer programming multiplies with an increase in the problem size, so the optimization of the steelmaking casting production process scheduling problems is needed according to the actual steelmaking situations, which is the primary motive of this research.

\section{PROBLEM DESCRIPTION}

\subsection{Problem analysis}

Most of the existing literature on steel rolling integration only considers the three processes of steelmaking, continuous casting and the hot rolling process. When the steelworks make the production plan, they must consider the condition of the production equipment, the weight and size of steel products, the composition and temperature of molten steel and molten iron. However, the composition and exact temperature of the molten steel cannot be identified before making the plan. The related information about the composition and temperature of the molten steel can only be driven according to the requirement of the steel products, a further setback in the control of the steelmaking process. In the actual production process, hot metal in the tundish package composition, temperature and other factors all affect the steelmaking process directly, which further affect the continuous casting and hot rolling processes. Therefore, this planning method cannot guarantee the lowest cost of production as a whole.

As a simple example, assume the next production batch plan includes a 100 ton plate (P1) and 100 tons of sheet (P2). The demand varieties of molten steels are respectively S1 and S2. Now the available molten iron for I1 and I2 are 100 tons each. Specifications of parameters 
are shown in Table I. From the data in Table I, the ingredient and the temperature of the molten iron I1 and molten steel S1 are relatively the same, as are I2 and S2. Under the premise of only considering the carbon content and temperature, and the line of production arrangement is designed as I2-S2-P2 I1-S1-P1, the total production cost will be the most economical.

Table I: Sample data.

\begin{tabular}{|c|c|c|c|}
\hline Project & Weight (ton) & Carbon content $(\%)$ & Temperature $\left({ }^{\circ} \mathrm{C}\right)$ \\
\hline P1 & 100 & 6 & 25 \\
\hline P2 & 100 & 2 & 25 \\
\hline S1 & 100 & 6 & 1650 \\
\hline S2 & 100 & 2 & 1520 \\
\hline I1 & 100 & 13 & 1435 \\
\hline I2 & 100 & 8 & 1342 \\
\hline
\end{tabular}

However, in the actual production, a dozen various hot metal ladles could possibly be used, several kinds of steel products could be used for production, and molten iron and steel components are complex and diverse, all of which greatly increase the difficulty of finding the optimal solution. However, solving this problem would be greatly economically beneficial. So, in the steel rolling integration decision issue, it is useful to consider the molten iron scheduling before the steelmaking process begins. This paper researches molten iron tank bag, steelmaking, continuous casting and hot rolling process of the steel rolling integration issue, establishes an optimization model and designs an optimized algorithm. In the end, a set of examples shows the solving process, and the performance of the algorithm is tested.

Because the converter steelmaking process is very complex, this paper does not consider the specific chemical reaction process, and concentrates on the steelmaking furnace plan. In addition, because of the difficulty of the hot roller material steel in continuous casting with the main materials of steel, the proportion of hot wire roller in the rolling element is very small, and the warm-up materials of multiple rolling units can be cast in advance by the temperature loading, so the hot roller material part is not considered in the preparation of steel rolling integrated batch planning.

\subsection{Modelling solutions}

In this paper, suit is identified as the minimum operation object for the tuple steelmaking, continuous casting and hot rolling process, and refers to the collection with the same level of steel grade, the same carbon content, the same temperature and the same casting width and thickness of the casting, thickness of the same width of rolling, and having roughly the same priority and date of delivery.

The strategies based on the integration are as follows: identify a single integration plan according to the match of casting machines and rolling machines, and ensure that it contains the number of casting units and rolling plans. Combine the slabs according to the effective furnace volume to form the units and then form the batch plan. Additionally, take unit groups as the basic operation subject to steelmaking, continuous casting and hot rolling and consider the constraints of continuous casting and hot rolling systematically. Then formulate the casting and rolling plan at the same time.

\subsection{The constraints satisfaction optimization model}

Considering slabs as visiting clients, batch plans as vehicles, and the unit casting length and main body casting length as a generalized time, models of Vehicle Routing Problem with Soft 
Time Window (VRPSTW) can thus be built for finding the solution. Since this problem is constrained by various complex technologies, some of which cannot be described by mathematical models, Constraint Satisfaction, CS is used to flexibly build the constraints satisfaction optimization model. In addition, the visual slabs should be put into unit groups according to the effective furnace volume in order to form the constraints satisfied optimization model with unit groups as the basic operation subjects. It is important to note that the cost of hot metal smelting steel should be included except for the melting pot weight when considering the converter steelmaking process.

\subsection{Model symbols}

The symbols and their interpretations in this model are shown in Table II.

Table II: Symbols and their interpretations.

\begin{tabular}{|c|l|l|}
\hline No. & Symbol & Interpretation \\
\hline 1 & $I$ & Suits set, $I=\left\{1,2, \ldots, i, \ldots, N_{I}\right\}$ \\
\hline 2 & $R$ & Converter planning set, $R=\left\{1,2, \ldots, r, \ldots, N_{R}\right\}$ \\
\hline 3 & $U$ & Cast planning set, $U=\left\{1,2, \ldots, u, \ldots, N_{U}\right\}$ \\
\hline 4 & $V$ & Rolling planning set, $V=\left\{1,2, \ldots, v, \ldots, N_{V}\right\}$ \\
\hline 5 & $S$ & Steel-making continuous-casting hot-rolling set, $S=\left\{1,2, \ldots, s, \ldots, N_{S}\right\}$ \\
\hline 6 & $W_{i}^{s}$ & Casting width of suit $i$, in meters \\
\hline 7 & $T_{i}^{s}$ & Casting thickness of suit $i$, in meters \\
\hline 8 & $W_{i}^{c}$ & Rolling width of suit $i$, in meters \\
\hline 9 & $T_{i}^{c}$ & Rolling thickness of suit $i$, in meters \\
\hline 10 & $C_{i}^{a}$ & Carbon content of molten steel of suit $i$, in percent \\
\hline 11 & $H_{i}^{a}$ & Molten steel temperature of suit $i$, in degrees \\
\hline 12 & $Q_{i}$ & Weight of suit $i$, in tons \\
\hline 13 & $Q_{i}^{\text {min }}$ & Minimum weight of the suit $i$, in tons \\
\hline 14 & $Q_{i}^{\text {max }}$ & Maximum weight of the suit $i$, in tons \\
\hline 15 & $L_{i}$ & Casting length of suit $i$, in meters \\
\hline 16 & $L_{i}^{\text {min }}$ & Allowed minimum casting length of suit $i$, in meters \\
\hline 17 & $L_{i}^{\text {max }}$ & Allowed maximum casting length of suit $i$, in meters \\
\hline 18 & $R_{i}^{c}$ & Casting hardness of suit $i$, dimensionless \\
\hline 19 & $S G_{i}$ & Steel grade of suit $i$, dimensionless \\
\hline 20 & $D_{i}$ & Delivery date of suit $i$, dimensionless \\
\hline 21 & $p_{i}$ & Priority of suit $i$, boolean variable, high priority $p_{i}=1$, low priority $p_{i}=0$ \\
\hline 22 & $D^{C}$ & $\begin{array}{l}\text { Allowed maximum change of carbon content in furnace planning for } \\
\text { adjacent suits, dimensionless }\end{array}$ \\
\hline 23 & $D^{H}$ & $\begin{array}{l}\text { Allowed maximum change of steel temperature in furnace planning for } \\
\text { adjacent suits, dimensionless }\end{array}$ \\
\hline 24 & $D^{S G}$ & $\begin{array}{l}\text { Allowed maximum change of steel grade in casting planning for adjacent } \\
\text { suits, dimensionless }\end{array}$ \\
\hline 25 & $D^{M T}$ & $\begin{array}{l}\text { Allowed maximum change of moulds applied in casting planning for } \\
\text { adjacent suits, dimensionless }\end{array}$ \\
\hline 26 & $D^{W}$ & $\begin{array}{l}\text { Allowed maximum change of width in rolling planning for adjacent suits, } \\
\text { dimensionless }\end{array}$ \\
\hline
\end{tabular}




\begin{tabular}{|c|c|l|}
\hline 27 & $D^{T}$ & $\begin{array}{l}\text { Allowed maximum change of thickness in rolling planning for adjacent } \\
\text { suits, dimensionless }\end{array}$ \\
\hline 28 & $D^{R}$ & $\begin{array}{l}\text { Allowed maximum change of hardness in rolling planning for adjacent } \\
\text { suits, dimensionless }\end{array}$ \\
\hline 29 & $D^{d}$ & $\begin{array}{l}\text { Allowed maximum change of delivery date in rolling planning for } \\
\text { adjacent suits, dimensionless }\end{array}$ \\
\hline 30 & $R L_{i}^{W}$ & $\begin{array}{l}\text { The maximum rolling length for the suit with the rolling width of } W_{i}^{c}, \text { in } \\
\text { meters }\end{array}$ \\
\hline 32 & $x_{s r u v i}$ & $\begin{array}{l}\text { Decision variable, } x_{\text {sruvi }}=1 \text { if suit } i \text { belongs to the } s \text { steel-making } \\
\text { continuous-casting hot-rolling, } r \text { furnace, } u \text { casting and } v \text { rolling planning, } \\
\text { otherwise, } x_{\text {sruvi }}=0\end{array}$ \\
\hline 33 & $z_{s u i j}$ & $\begin{array}{l}\text { Decision variable, } y_{s r i j}=1 \text { if the suit } j \text { is after the suit } i \text { in } r \text { furnace } \\
\text { planning, otherwise, } y_{\text {srij }}=0\end{array}$ \\
\hline 34 & $w_{s v i j}$ & $\begin{array}{l}\text { Decision variable, } z_{\text {suij }}=1 \text { if the suit } j \text { is after the suit } i \text { in } s \text { steel-making } \\
\text { continuous-casting hot-rolling, } u \text { casting, otherwise, }, z_{\text {suij }}=0\end{array}$ \\
\hline $\begin{array}{l}\text { Decision variable, } w_{\text {svij }}=1 \text { if the suit } j \text { is after the suit } i \text { in } s \text { steel-making } \\
\text { continuous-casting hot-rolling, } v \text { rolling planning, otherwise, } w_{s v i j}=0\end{array}$ \\
\hline
\end{tabular}

\subsection{Modelling}

The model of steelmaking continuous casting hot rolling planning is established based on the VRPSTW problem modelling:

$$
\begin{aligned}
& \min Z=\alpha_{1}\left(M-\sum_{s=1}^{N_{S}} \sum_{r=1}^{N_{R}} \sum_{u=1}^{N_{U}} \sum_{v=1}^{N_{V}} \sum_{i=1}^{N_{I}} c_{i} \cdot x_{s r u v i}\right)+\alpha_{2}\left(N_{S}\right)+\alpha_{3} \sum_{s=1}^{N_{S}} \sum_{r=1}^{N_{R}} \sum_{i=1}^{N_{I}} \sum_{j=1}^{N_{I}} c_{i j}^{R} \cdot y_{s r i j} \\
& +\alpha_{4} \sum_{s=1}^{N_{S}} \sum_{u=1}^{N_{U}} \sum_{i=1}^{N_{I}} \sum_{j=1}^{N_{I}} c_{i j}^{U} \cdot z_{\text {suij }}+\alpha_{5} \sum_{s=1}^{N_{S}} \sum_{v=1}^{N_{V}} \sum_{i=1}^{N_{I}} \sum_{j=1}^{N_{I}} c_{i j}^{V} \cdot w_{s v i j}+\alpha_{6} \sum_{s=1}^{N_{S}} \sum_{r=1}^{N_{R}} \sum_{u=1}^{N_{U}} \sum_{v=1}^{N_{V}} \sum_{i=1}^{N_{I}} q_{i} \cdot\left(1-x_{\text {sruvi }}\right) \text {, } \\
& \text { s.t. } Q_{\min } \leq \sum_{i=1}^{N_{R}}\left(x_{\text {sruvi }} \cdot Q_{i}\right) \leq Q_{\max }, \forall s, r, u \\
& \sum_{i=1}^{N_{R}} \sum_{j=1}^{N_{R}} z_{\text {suij }} \leq D^{M T} \text {, if } z_{\text {suij }}=1 \text { and }\left(\left|W_{i}^{s}-W_{j}^{s}\right|>0 \text { or }\left|T_{i}^{s}-T_{j}^{s}\right|>0\right), \forall s, r, u \\
& \left|C_{i}-C_{j}\right| \leq D^{C} \text { and }\left|H_{i}-H_{j}\right| \leq D^{H} \text { and }\left|D_{i}-D_{j}\right| \leq D^{d} \text {, if } y_{s r i j}=1, \forall s, r \\
& \left|S G_{i}-S G_{j}\right| \leq D^{S G} \text { and }\left|D_{i}-D_{j}\right| \leq D^{d} \text {, if } z_{\text {suij }}=1, \forall s, u \\
& \left|W_{i}^{C}-W_{j}^{C}\right| \leq D^{W} \text { and }\left|T_{i}^{C}-T_{j}^{C}\right| \leq D^{T} \text { and }\left|R_{i}^{C}-R_{j}^{C}\right| \leq D^{R} \text { and }\left|D_{i}-D_{j}\right| \leq D^{d} \text {, if } w_{s v i j}=1 \text {, } \\
& \sum_{i=1}^{N_{R}} x_{\text {sruvi }} \cdot L_{i} \leq L_{i}^{\max }, \text { if } x_{\text {sruvi }}=1 \text { and } W_{i}^{C}=W_{i^{\prime}}^{C}, \forall v, i, i^{\prime} \\
& L_{i}^{\min } \leq \sum_{i=1}^{N_{R}}\left(\sum_{r=1}^{N_{R}} \sum_{u=1}^{N_{U}} x_{\text {sruvi }} \cdot L_{i}\right) \leq L_{i}^{\max }, \forall s, v \\
& \sum_{s}^{N_{S}} \sum_{r}^{N_{R}} \sum_{u}^{N_{U}} \sum_{v}^{N_{V}} x_{\text {sruvi }} \leq 1, \quad \forall i \\
& x_{s r u v i}, y_{s r i j}, z_{s u i j}, w_{s v i j} \in\{0,1\}, \quad \forall s, r, u, v, i, j
\end{aligned}
$$


Eq. (1) is the objective function which aims to maximize profit, as well as minimize the numbers of plans, steelmaking penalty function values, continuous casting penalty function values and loss function values. $\alpha$ represents the weight of each objective. $c_{i}$ and $q_{i}$ represent the profit function with the suit in the planning and the loss function without the suit respectively. If suit $j$ is after suit $i$, then the penalty function of the steelmaking, continuous casting and rolling are expressed as $c_{i j}{ }^{R}, c_{i j}{ }^{U}$ and $c_{i j}{ }^{V}$ respectively.

Eqs. (2) to (10) are constraint conditions. Eq. (2) represents the allowed minimum (maximum) tundish weight. Eq. (3) represents change modulus in cast. Eq. (4) represents the allowed maximum changes in terms of the carbon content of the steel, the steel temperature and delivery time between the adjacent suits in furnace. Eq. (5) represents the allowed maximum changes in terms of steel grade and delivery date between the adjacent suits in the cast planning. Eq. (6) represents the allowed maximum changes in terms of width, thickness, hardness and delivery time between the adjacent suits in the rolling planning. Eq. (7) represents the length limit with the same wide rolling. Eq. (8) represents the allowed the minimum (maximum) length in rolling unit. Eq. (9) represents one suit which can only be arranged to one planning. Eq. (10) represents the scopes of the related variables.

\section{FUZZY GENETIC ALGORITHM}

The model of the integrated batch planning process based VRPSTW is one of combinational optimization problems, also is NP-hard problem, so it is critical to design an effective algorithm for this model. An improved fuzzy genetic algorithm is proposed to obtain the best possible solution in limited time.

This study proposes an adaptive fuzzy logic-based genetic approach for the detection of integrated batch planning process. As the approach's major novelty, an adaptive-fuzzy logic module is integrated with the conventional GA to improve the performance of the GA and reduce the premature convergence problem by adjusting the algorithm's parameters. The flow chart of fuzzy logic genetic algorithm is shown in Fig. 1.

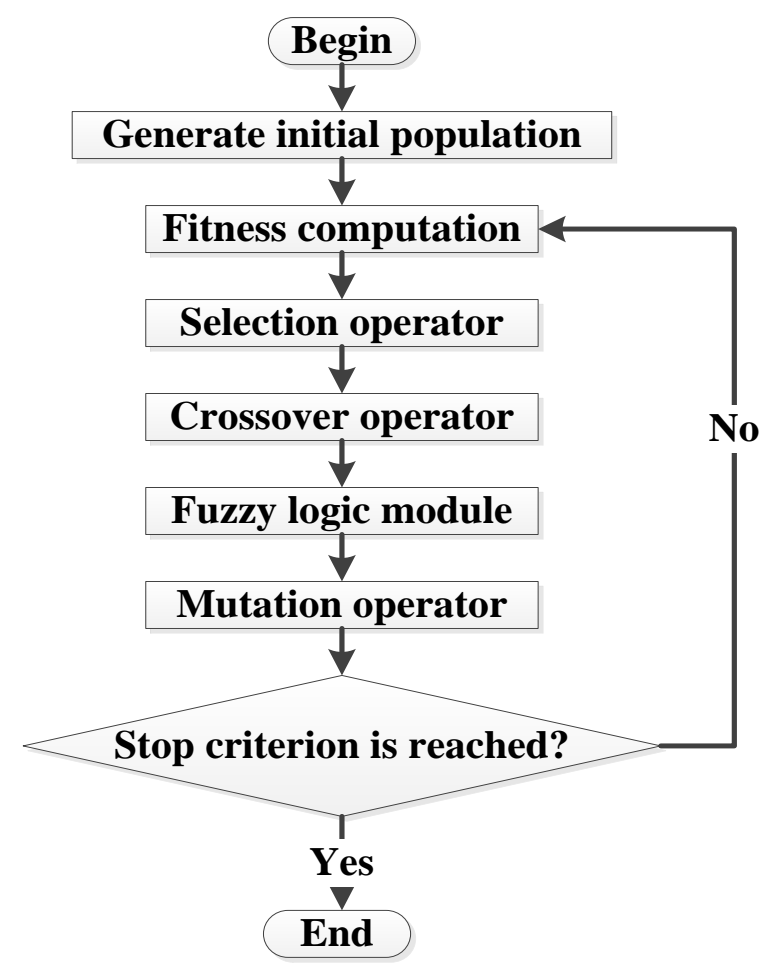

Figure 1: The flow chart of fuzzy logic genetic algorithm. 


\subsection{Design of GA}

\section{(1) Encoding}

The encoding of the integrated batch planning process includes several decision variables such as integration of steel rolling batch planning, furnace, time, cast and rolling planning. Therefore, a matrix encoding method which is similar to the particle swarm optimization algorithm is employed in this paper, as shown below:

$$
A_{4 \times N_{I}}=\left[\begin{array}{cccc}
a_{11} & a_{12} & \cdots & a_{1 N_{I}} \\
a_{21} & a_{22} & \cdots & a_{2 N_{I}} \\
a_{31} & a_{32} & \cdots & a_{3 N_{I}} \\
a_{41} & a_{42} & \cdots & a_{4 N_{I}}
\end{array}\right]
$$

where: $\left(a_{11}, a_{12}, \ldots, a_{1 N I}\right)$ represents the integrated batch planning, $a_{1 i} \in\left\{1,2, \ldots, N_{S}\right\}$, $i \in\left\{1,2, \ldots, N_{I}\right\}$, and each column corresponds with the suit; i.e., $a_{12}=6$ means that the suit 2 belongs to the 6 integrated batch planning. $\left(a_{21}, a_{22}, \ldots, a_{2 N I}\right)$ represents the furnace planning, $a_{2 i} \in\left\{1,2, \ldots, N_{R}\right\}, i \in\left\{1,2, \ldots, N_{I}\right\} .\left(a_{31}, a_{32}, \ldots, a_{3 N I}\right)$ represents the casting planning, $a_{3 i} \in\left\{1,2, \ldots, N_{U}\right\}, i \in\left\{1,2, \ldots, N_{I}\right\} .\left(a_{41}, a_{42}, \ldots, a_{4 N I}\right)$ represents the rolling planning, $a_{4 i} \in\left\{1,2, \ldots, N_{V}\right\}, i \in\left\{1,2, \ldots, N_{I}\right\}$.

(2) Fitness function

Fitness function is the inverse of the objective function.

(3) Selection operator

In this binary tournament selection process, two individuals are selected at random from the population and the fittest one is selected for reproduction.

(4) Crossover operator

Hierarchical order crossover operator is used for the matrix encoding method. The basic rule of hierarchical order crossover operator is execution ordered crossover operation separately for each line. The order crossover operator is designed for order-based permutation problems. Two crossover points are randomly selected and the segment between them is copied to the offspring from the first parent. Starting from the second crossover point in the second parent, the elements are copied to the offspring in the order they appear in the second parent, avoiding repetition. The second offspring is created in the same way, reversing the roles of the parents.

(5) Mutation operator

Similar to the crossover operator, the mutation operator is hierarchical inverted mutation operator. This operator works by randomly selecting two positions in the string and reversing the order in which the values appear between those positions.

\subsection{Adaptive-fuzzy logic module}

The fuzzy logic module is composed of fuzzification, fuzzy inference and clarifications. This paper proposes, under the consideration that the fitness value of the chromosome has an impact on the natural evolution, an adaptive-fuzzy logic module which could adjust mutation probability according to the fitness value of the chromosome and the average fitness value of the population. The idea is as follows: (1) Improve the mutation probability to eliminate low fitness values of individuals when there is a significant difference between the fitness value of the current chromosome and the highest fitness value of the chromosome. Otherwise, lower mutation probability. (2) Improve the mutation probability to avoid the premature convergence when the range of the average fitness value is small. Otherwise, lower mutation probability. 
$F$ is the fitness value of the current chromosome. $\bar{F}_{r}$ is the average fitness value of chromosomes of generation $r$ 's population. $F_{\max }$ is the fitness value of the best chromosome in the current population. $F_{\min }$ is the fitness value of the worst chromosome in the current population. The output parameter is the mutation probability, and the input parameters are shown in Table III.

Table III: Input parameters of fuzzy logic module.

\begin{tabular}{|c|l|}
\hline Input parameter & \multicolumn{1}{|c|}{ Parameter explanation } \\
\hline$\Delta \bar{F}$ & The range of the average fitness value, $\Delta \bar{F}=\frac{\left(\overline{F_{r}}-\overline{F_{r-1}}\right)}{\overline{F_{r-1}}}, \Delta \bar{F} \in[0,1], r>1$ \\
\hline$\alpha$ & $\begin{array}{l}\text { The gap of the fitness value between the current chromosome and the } \\
\text { best chromosome, } \alpha=\frac{\left(F_{\max }-F\right)}{\left(F_{\max }-F_{\min }\right)}, \alpha \in[0,1]\end{array}$ \\
\hline
\end{tabular}

There are nine sematic values in the fuzzy logic module, where ES means extremely small, VS means very small, $\mathrm{S}$ means small, RS means relatively small, $\mathrm{M}$ means medium, RL means relatively larger, L means larger, VL means very larger and EL means extremely larger. This paper uses the triangle membership function. The graphic of membership function, shown in Fig. 2, is derived according to the sematic value and triangle membership function.

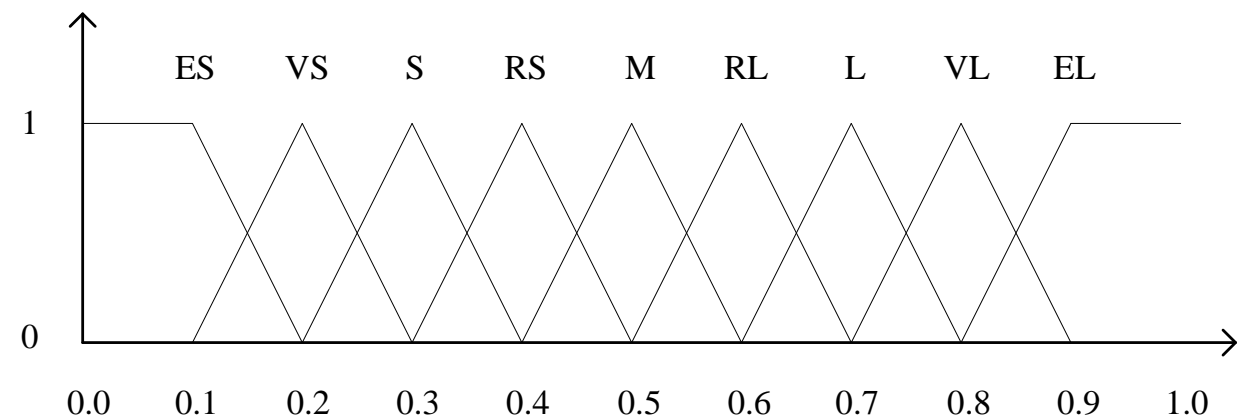

Figure 2: The membership function graphic of input and output parameter.

The fuzzy logic rules, shown in Table IV, are derived according to the fuzzy logic and the membership.

Table IV: The fuzzy logic rules of mutation probability.

\begin{tabular}{|c|c|c|c|c|c|c|c|c|c|}
\hline$\Delta \vec{F}(t)$ & ES & VS & S & RS & M & RL & L & VL & EL \\
\hline ES & M & RL & RL & L & L & VL & VL & EL & EL \\
\hline VS & RS & M & RL & RL & L & L & VL & VL & EL \\
\hline S & RS & RS & M & RL & RL & L & L & VL & VL \\
\hline RS & S & RS & RS & M & RL & RL & L & L & VL \\
\hline $\mathbf{M}$ & S & S & RS & RS & M & RL & RL & L & L \\
\hline RL & VS & S & S & RS & RS & M & RL & RL & L \\
\hline L & VS & VS & S & S & RS & RS & M & RL & RL \\
\hline VL & ES & VS & VS & S & S & RS & RS & M & RL \\
\hline EL & ES & ES & VS & VS & S & S & RS & RS & M \\
\hline
\end{tabular}




\section{NUMERICAL SIMULATION}

\subsection{Design of the numerical simulation}

Matlab 2012R is employed for the numerical study, and the data are collected from a steel company in China. The data are named as group A, group B and group $\mathrm{C}$ which include orders over a period of three months.

The main process parameters are as follows: the enterprise has four similar converters, two similar continuous casting machines and one hot rolling mill. According to machine capacity matching calculation, the steel rolling integration plan can be identified which contains four furnace plans, four pouring time plans and four rolling units. The valid capacity of the converter furnace is $280 \mathrm{t} \sim 300 \mathrm{t}$. The minimum weight of the continuous caster tundishis is $Q_{\min }=1000 \mathrm{t}$, and the maximum weight is $Q_{\max }=2700 \mathrm{t}, D^{M T}=1$. Hot rolling mill: $L_{\min }=45000 \mathrm{t}, L_{\max }=70000 \mathrm{~m}, R L_{i}{ }^{W}=20000 \mathrm{~m}$. The parameters of fuzzy genetic algorithm are: population size Popsize $=300$, maximum number of optimization $T_{\max }=5000$.

\subsection{Results and analysis of the numerical simulation}

The results of the numerical study are shown in Table V, where $N_{s}$ represents the number of the integrated batch planning process planning; $B R=$ (weight for the orders included in the plan/total weight for all orders $) \times 100 \%$; order matching ratio $M O R$ is the order matching for the suits before and after processing. If the continuous casting machine processing order for the slab is $\lambda$, and its processing sequence are $\lambda$ on hot rolling mill, then the order matching the ratio of this slab is $100 \%$; NOR = (invalid loading weight/total weight for all orders $) \times 100 \%$; $O T D R=($ weight of orders delivered on time/total weight for all the orders $) \times 100 \% ; A C H=$ total weight of smelting/number of furnace planning; $A C W=$ total casting weight/number of cast planning; $A R L=$ total rolling length/rolling units; $A C T A$ is the average time for the calculation of the integrated batch planning optimization. The values of carbon content and tapping temperature are set as fixed values, and in the actual production process the two parameters are usually set in value ranges.

Table V: The results of the integrated batch planning.

\begin{tabular}{|c|c|c|c|c|c|c|c|c|c|}
\hline Project & $\boldsymbol{N}_{\boldsymbol{s}}$ & $\boldsymbol{B R}$ & $\boldsymbol{M O R}$ & $\boldsymbol{N O R}$ & $\boldsymbol{O T D R}$ & $\boldsymbol{A C H}$ & $\boldsymbol{A C W}$ & $\boldsymbol{A R L}$ & $\boldsymbol{A C T}$ \\
\hline Unit & - & $\%$ & $\%$ & $\%$ & $\%$ & $\mathrm{t}$ & $\mathrm{t}$ & $\mathrm{m}$ & $\mathrm{min}$ \\
\hline A & 123 & 97.8 & 99.1 & 0.13 & 96.5 & 295 & 2498 & 62310 & 0.15 \\
\hline B & 158 & 98.5 & 99.2 & 0.15 & 95.8 & 296 & 2630 & 66782 & 0.19 \\
\hline C & 142 & 98.2 & 98.7 & 0.20 & 96.7 & 292 & 2587 & 64253 & 0.17 \\
\hline Average & - & 98.2 & 99.0 & 0.16 & 96.33 & 294.33 & 2572 & 64448 & 0.17 \\
\hline
\end{tabular}

Analysis of the numerical study:

(1) The results of the numerical study show that the proposed improved fuzzy genetic algorithm can improve the $B R$ as high as $98.2 \%$, as well as decrease the NOR $(0.16 \%)$ and increase the $O T D R(96.33 \%)$.

(2) Based on the working procedure, fuzzy genetic algorithm calculates the average data of steel weight, and continuous casting weight and length of rolling are respectively $294.33 \mathrm{t}$, $2572 \mathrm{t}$ and $64448 \mathrm{~m}$. Such a process plan can give full play to the maximum capacity of each process. In addition, fuzzy genetic algorithm was used to obtain the matching rate of arrangement which is up to $99.0 \%$. The matching and coordination can be fully realized between each process, and the waiting time between the various processes could be greatly reduced. It could improve the overall production efficiency, and the management of the production level of enterprises. 


\section{CONCLUSIONS}

Optimization of scheduling of the integrated batch planning process can reduce energy consumption and production costs, while at the same time increasing profit, so many steel companies are focused on how to optimize the integrated batch planning process. This paper establishes the model of an integrated batch planning process, and proposes the improved algorithms for this problem. The simulation results of a computerized scheduling system are also given to prove the model. It has been proved that the proposed improved fuzzy genetic algorithm is a very efficient and effective approach and it can help companies obtain scheduling results in less overall computing time and experience better performances for the integrated batch planning process scheduling problem. This steel rolling batch plan has the further benefit of reducing the amount of the default order, thus improving production efficiency and market competitiveness. In addition, the average calculation time is only 0.17 minutes, which can solve the large-scale problems encountered in the process of production in an efficient amount of time, and could achieve fast and effective connection with the manufacturing execution system.

\section{ACKNOWLEDGEMENT}

This work was supported by the Hainan Province Natural Science Fund (No. 20151003 and No. 20156233).

\section{REFERENCES}

[1] Bellabdaoui, A.; Teghem, J. (2006). A mixed-integer linear programming model for the continuous casting planning, International Journal of Production Economics, Vol. 104, No. 2, 260-270, doi:10.1016/j.ijpe.2004.10.016

[2] Pan, Q.-K., Wang, L.; Mao, K.; Zhao, J.-H.; Zhang, M. (2013). An effective artificial bee colony algorithm for a real-world hybrid flowshop problem in steelmaking process, IEEE Transactions on Automation Science and Engineering, Vol. 10, No. 2, 307-322, doi:10.1109/ tase.2012.2204874

[3] Atighehchian, A.; Bijari, M.; Tarkesh, H. (2009). A novel hybrid algorithm for scheduling steel-making continuous casting production, Computers \& Operations Research, Vol. 36, No. 8, 2450-2461, doi:10.1016/j.cor.2008.10.010

[4] Liu, W.; Sun, L.-L. (2012). Steel-making and continuous/ingot casting scheduling of mixed charging plan based on batch splitting policy, International Journal of Iron and Steel Research, Vol. 19, No. 2, 17-21, doi:10.1016/s1006-706x(12)60054-5

[5] Pacciarelli, D.; Pranzo, M. (2004). Production scheduling in a steelmaking-continuous casting plant, Computers \& Chemical Engineering, Vol. 28, No. 12, 2823-2835, doi:10.1016/ j.compchemeng.2004.08.031

[6] Li, J.; Xiao, X.; Tang, Q.-H.; Floudas, C. A. (2012). Production scheduling of a large-scale steelmaking continuous casting process via unit-specific event-based continuous-time models: short-term and medium-term scheduling, Industrial \& Engineering Chemistry Research, Vol. 51, No. 21, 7300-7319, doi:10.1021/ie2015944

[7] Assaf, I.; Chen, M.; Katzberg, J. (1997). Steel production schedule generation, International Journal of Production Research, Vol. 35, No. 2, 467-477, doi:10.1080/002075497195858

[8] Tang, L.-X.; Liu, J.-Y.; Rong, A.-Y.; Yang, Z.-H. (2001). A review of planning and scheduling systems and methods for integrated steel production, European Journal of Operational Research, Vol. 133, No. 1, 1-20, doi:10.1016/s0377-2217(00)00240-x

[9] Sun, F.-Q.; Zheng, B.-L.; Cui, J.-J.; Wang, D.-W.; Liu, X.-S. (2000). The research of production planning of integrated management in steelmaking-hot rolling, Acta Automatica Sinica, Vol. 26, No. 3, 409-413 (in Chinese) 
[10] Cowling, P.; Rezig, W. (2000). Integration of continuous caster and hot strip mill planning for steel production, Journal of Scheduling, Vol. 3, No. 4, 185-208, doi:10.1002/10991425(200007/08)3:4<185::aid-jos42>3.0.c0;2-g

[11] Zhu, B.-L.; Yu, H.-B.; Huang, X.-Y. (2006). Research approaches on integrated planning for iron and steel enterprises, Proceedings of the IEEE International Conference on Systems, Man and Cybernetics (SMC'06), 5024-5029, doi:10.1109/icsmc.2006.385104

[12] Li, Y.-H.; Hu, G.-F.; Wang, W.; Ning, S.-S. (2005). Research on the method of SM-CC-HR integrated production planning, Control Engineering of China, Vol. 12, No. 6, 549-552 (in Chinese)

[13] Liang, H.-L.; Li, S.-J. (2008). CC-DHCR planning and scheduling method based on slab cluster, Journal of Advanced Manufacturing Systems, Vol. 7, No. 2, 249-252, doi:10.1142/ s0219686708001437

[14] Gao, H.-M.; Zeng, J.-C.; Sun, G.-J. (2002). Multi-agent approach for planning and scheduling of integrated steel processes, Proceedings of the IEEE International Conference on Systems, Man and Cybernetics (SMC'02), Vol. 6, 6 pages, doi:10.1109/icsmc.2002.1175626

[15] Ma, T.-M.; Zhang, L.; Hu, G.-F.; Zheng, B.-L.; Cai, T.-Y. (2005). New technology of integrated batch planning (IBP), Control Engineering of China, Vol. 12, No. 6, 533-536 (in Chinese)

[16] Xu, J.; Lv, Z.-M.; Xu, J.-W. (2006). Model and algorithm of integrative batch planning based on parallel strategy for steelmaking-continuous casting-hot rolling, Control and Decision, Vol. 21, No. 9, 979-983 (in Chinese)

[17] Sun, L.; Li, T.-K. (2007). Constraint satisfaction algorithm of batch planning for steelmaking-continuous casting-hot rolling, Computer Integrated Manufacturing Systems, Vol. 13, No. 5, 940-944 (in Chinese)

[18] Lin, X.-X.; Floudas, C. A.; Modi, S.; Juhasz, N. M. (2002). Continuous-time optimization approach for medium-range production scheduling of a multiproduct batch plant, Industrial \& Engineering Chemistry Research, Vol. 41, No. 16, 3884-3906, doi:10.1021/ie011002a

[19] Janak, S. L.; Floudas, C. A.; Kallrath, J.; Vormbrock, N. (2006). Production scheduling of a large-scale industrial batch plant. I. Short-term and medium-term scheduling, Industrial \& Engineering Chemistry Research, Vol. 45, No. 25, 8234-8252, doi:10.1021/ie0600588

[20] Janak, S. L.; Floudas, C. A.; Kallrath, J.; Vormbrock, N. (2006). Production scheduling of a large-scale industrial batch plant. II. Reactive scheduling, Industrial \& Engineering Chemistry Research, Vol. 45, No. 25, 8253-8269, doi:10.1021/ie0600590

[21] Chen, H.-X.; Chu, C.-B.; Proth, J.-M. (1998). An improvement of the Lagrangean relaxation approach for job shop scheduling: a dynamic programming method, IEEE Transactions on Robotics and Automation, Vol. 14, No. 5, 786-795, doi:10.1109/70.720354

[22] Fisher, M. L. (1981). The Lagrange relaxation method for solving integer programming problems, Management Science, Vol. 27, No. 1, 1-18, doi:10.1287/mnsc.27.1.1

[23] Tang, L.-X.; Liu, J.-Y.; Rong, A.-Y.; Yang, Z.-H. (2000). A mathematical programming model for scheduling steelmaking-continuous casting production, European Journal of Operational Research, Vol. 120, No. 2, 423-435, doi:10.1016/s0377-2217(99)00041-7

[24] Luh, P. B.; Wang, J. H.; Wang, J. L.; Tomastik, R. N.; Howes, T. D. (1997). Near-optimal scheduling of manufacturing systems with presence of batch machines and setup requirements, CIRP Aunnals - Manufacturing Technology, Vol. 26, No. 1, 397-402, doi:10.1016/s0007$\underline{8506(07) 60851-8}$

[25] Hoitomt, D. J.; Luh, P. B.; Pattipati, K. R. (1993). A practical approach to job shop scheduling problems, IEEE Transactions on Robotics and Automation, Vol. 9, No. 1, 1-13, doi:10.1109/ $\underline{70.210791}$ 